\title{
MIT
}

\section{Photonic device sensitivity analysis methods: towards process variation-aware silicon photonics design}

The MIT Faculty has made this article openly available. Please share how this access benefits you. Your story matters.

\begin{tabular}{|l|l|}
\hline As Published & $10.1117 / 12.2529198$ \\
\hline Publisher & SPIE-Intl Soc Optical Eng \\
\hline \hline Version & Final published version \\
\hline Citable link & https://hdl.handle.net/1721.1/132246 \\
\hline Terms of Use & $\begin{array}{l}\text { Article is made available in accordance with the publisher's } \\
\text { policy and may be subject to US copyright law. Please refer to the } \\
\text { publisher's site for terms of use. }\end{array}$ \\
\hline
\end{tabular}




\section{Photonic device sensitivity analysis methods: towards process variation- aware silicon photonics design}

El-Henawy, Sally, Zhang, Zhengxing, Miller, Ryan, Boning, Duane

Sally I. El-Henawy, Zhengxing Zhang, Ryan Miller, Duane S. Boning, "Photonic device sensitivity analysis methods: towards process variationaware silicon photonics design," Proc. SPIE 11103, Optical Modeling and System Alignment, 111030P (11 September 2019); doi: 10.1117/12.2529198

EDent: SPIE Optical Engineering + Applications, 2019, San Diego, California, United States 


\title{
Photonic device sensitivity analysis methods: towards process variation-aware silicon photonics design
}

\author{
Sally I. El-Henawy*a ${ }^{*}$ Zhengxing Zhang ${ }^{\mathrm{a}}$, Ryan Miller ${ }^{\mathrm{b}}$, and Duane S. Boning ${ }^{\mathrm{a}}$

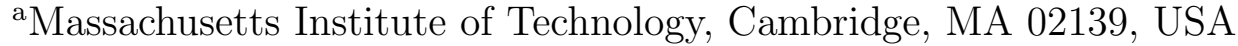 \\ ${ }^{\mathrm{b}}$ Coventor, Inc., 135 Beaver St. Suite 205, Waltham, MA 02452, USA
}

\begin{abstract}
Silicon photonics offers the ability to fabricate and integrate photonic and electronic components using existing integrated circuit fabrication infrastructure. Recent work seeks to understand the impact of IC process variations on performance of photonic components. In particular, methods for analysis that identify sensitivity of photonic components to process variations are crucial to enable viable design and manufacturing of silicon photonic systems.

We present two different and complementary methods for understanding the impact of geometric process variations on photonics components: ensemble statistical virtual fabrication simulations, and adjoint methods. These are utilized to identify the most sensitive regions of a Y-splitter photonic component to line edge roughness (LER) due to inherent lithography and etch process variations. In the ensemble approach, we simulate multiple instantiations with random LER applied to specific sections of the Y-splitter. This enables localization and quantification of LER impact on transmission, phase imbalance, and excess losses. These evaluations, however, come at the cost of many simulations. In adjoint sensitivity evaluation, only one or two simulations can identify regions most sensitive to LER. While first-order linear sensitivity is extracted, the adjoint has challenges in quantifying mean variation impacts. Both methods reveal that the Y-splitter is most sensitive to LER in the input taper, accounting for over $95 \%$ of the imbalance transmission. These two methods can be combined to quantify mean, variance, and sensitivity of photonic device components in the face of statistical variations. Incorporated into future photonic process design kits (PDKs), these analysis methods will help designers predict and optimize photonic component performance and yield.
\end{abstract}

Keywords: Silicon photonics, line edge roughness, adjoint method, process variations, Y-splitter, DFM, photonic devices, sensitivity analysis.

\section{INTRODUCTION}

The high material contrast in silicon photonics enables tight light confinement and high density integration, which in turn enables additional complexity and introduces more functionalities and applications that silicon photonics can support. ${ }^{1-4}$ This makes silicon photonics a promising design platform. To be cost-effective, silicon photonics leverages the existing complementary metal-oxide-semiconductor (CMOS) integrated circuit (IC) manufacturing infrastructure for fabricating photonic components. This means, however, that silicon photonics is susceptible to many of the same fabrication process variations as are experienced in CMOS IC manufacturing.

Unlike in CMOS ICs where the methods and tools are well developed to analyze and optimize circuit performance, manufacturability, and yield with respect to process variations, in silicon photonics relatively few methods have been reported and demonstrated to analyze the effect of process variations on photonic performance. ${ }^{5-8}$ The design of a silicon-photonics-based system is a time-consuming and slow process: the designer must often design at both the photonic component or device level, and at the photonic circuit level, typically focusing on consideration of interacting physical effects to achieve desired performance. After fabrication, however, the yield can be very low as process variation effects degrade performance. Such variation effects are typically not well considered during the design phase, due to the lack of mature models, design for manufacturability (DFM) methods, and simulation tools for photonic components that are compatible with the commercial CMOS process

*elhenawy@mit.edu

Optical Modeling and System Alignment, edited by Mark A. Kahan, José Sasián,

Richard N. Youngworth, Proc. of SPIE Vol. 11103, 111030P - () 2019 SPIE

CCC code: $0277-786 \mathrm{X} / 19 / \$ 21 \cdot$ doi: $10.1117 / 12.2529198$

Proc. of SPIE Vol. 11103 111030P-1 
steps. So, the need for mature variation-aware models and methods for silicon photonics becomes clear in order to achieve high-yield photonic integrated circuits (PICs).

In this paper, we present two different yet complementary methods for performing sensitivity analysis against geometric perturbations on photonics components: ensemble virtual fabrication simulations and the adjoint method. We use line edge roughness (LER) $)^{9}$ as a process variation example to demonstrate the methodology. LER is an inherent random process variations present in IC and photonic fabrication, causing perturbations to the geometry of the component and affecting its light guiding capability. However, some regions within a device can be more sensitive to these variations in geometry. An important question is understanding where and how LER impacts the device (e.g., to guide the designer in seeking to make the component more robust to such variation). To this end, we apply ensemble virtual fabrication simulations and adjoint methods. We find that the methods are consistent in identifying where the most sensitive regions of the device are, and combine to form a powerful methodology to predict device sensitivities and output variation. The same methodology can be applied on other process variations and photonic components, to localize and understand variation impact.

\section{ENSEMBLE SIMULATION}

We perform the sensitivity analysis for one of the commonly used photonic components which is present in most PICs, a silicon-on-insulator (SOI) Y-splitter. The geometry of the Y-splitter used, shown in Fig. 1a, is similar to the one presented in, ${ }^{10}$ with a nominal waveguide width of $500 \mathrm{~nm}$, length of $15 \mu \mathrm{m}$ and thickness of $220 \mathrm{~nm}$.

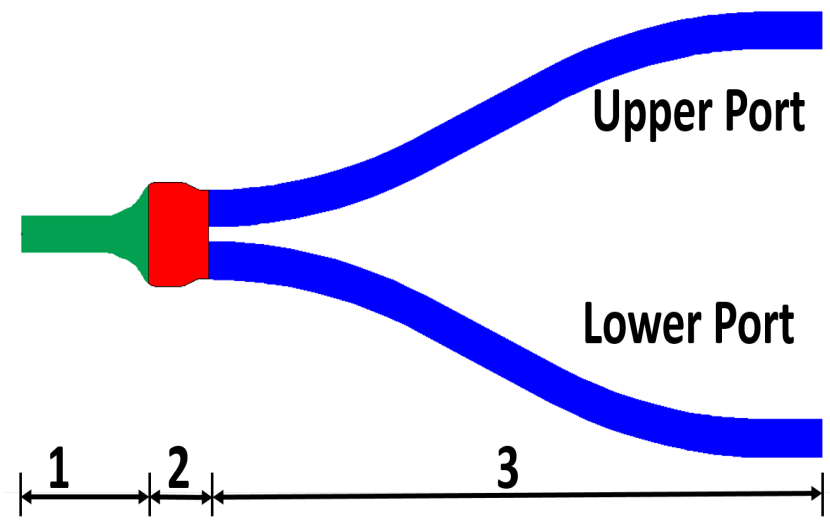

(a)

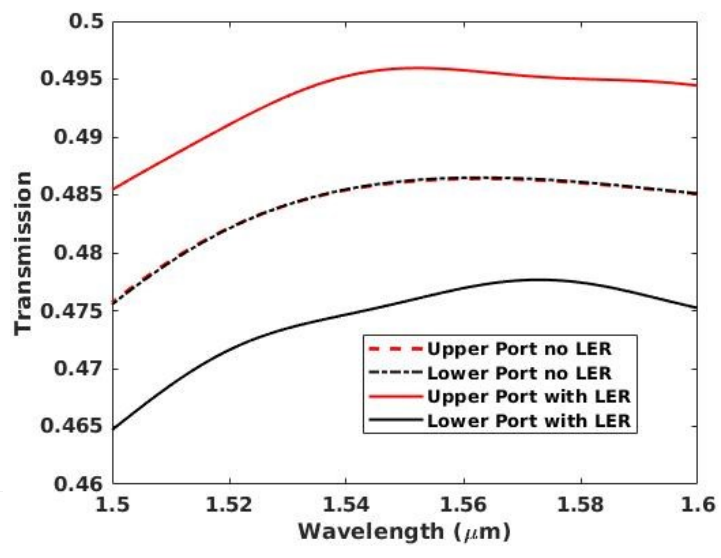

(b)

Figure 1: (a) Y-splitter geometry where region 1 (green) is the input taper, region 2 (red) is the junction and region 3 (blue) contains the output arms. (b) Normalized transmission for two cases: when no LER is present in the Y-splitter and when LER $\left(A=3 \mathrm{~nm}\right.$ and $\left.L_{c}=10 \mathrm{~nm}\right)$ is added to all sidewalls of the Y-splitter.

To study the sensitivity of the Y-splitter in the presence of LER, we virtually fabricate the Y-splitter by modeling the process steps using Coventor's SEMulator3D ${ }^{\circledR 11}$ software and introduce the LER during the lithography step. The LER is defined as Gaussian noise $N$ using a Fourier synthesis technique ${ }^{12,13}$ and is imposed to the Y-splitter as geometric width perturbations. The two parameters that characterize the LER are its root mean square amplitude $(A)$ and correlation length $\left(L_{c}\right)$ as in (1):

$$
N=A^{2} e^{-\left(x / L_{c}\right)^{2}}
$$

where $x$ is the distance along the length of the structure. The structures with LER imposed are then exported from SEMulator3D ${ }^{\circledR}$ and imported into Lumerical FDTD $^{14}$ for optical simulations.

Previous results ${ }^{6}$ showed that LER causes transmission imbalance between the two Y-splitter output ports as opposed to the expected balanced transmission in the ideal or nominal case, Fig. 1b. Running an ensemble 
of 50 different instantiations, as shown in Fig. 2, enables us to identify the statistical nature of LER impact by calculating the wavelength-dependent mean and standard deviation of the transmission imbalance $(\Delta T)$, defined as the absolute value of the difference in the normalized transmitted power between the two output ports, $\Delta T=\left|T_{\text {upper }}-T_{\text {lower }}\right|$ where $T_{\text {upper }}$ is the transmitted power in the upper output port normalized to the power at the input port. However, these simulations alone do not provide information about the region of the Y-splitter that is most sensitive to LER and contributes the most to the output variation (i.e., spatial sensitivity to LER).

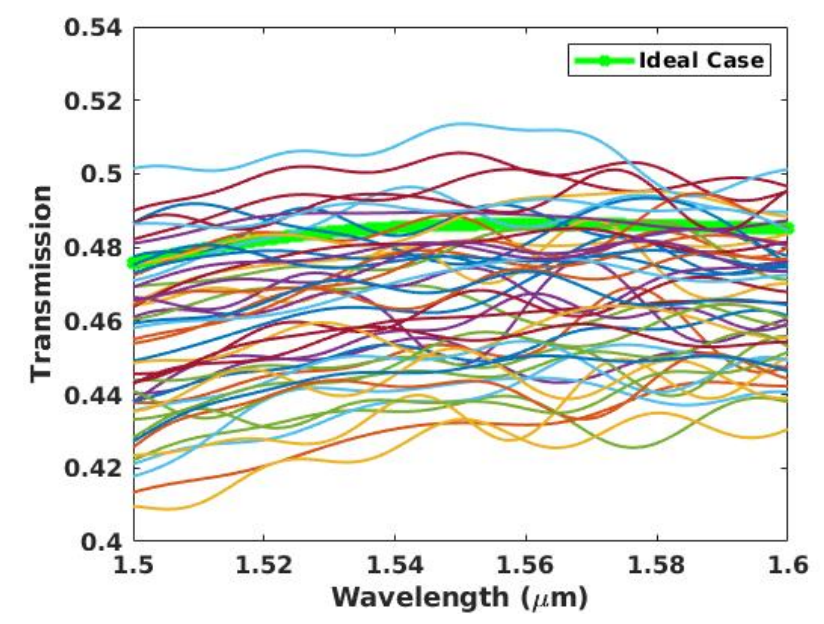

(a)

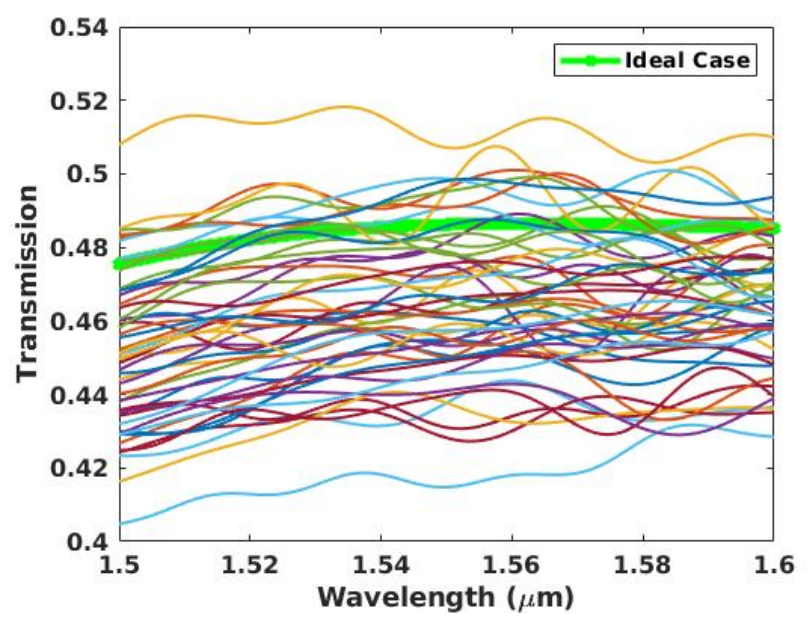

(b)

Figure 2: Normalized transmitted power for 50 different instantiations applying LER with $A=12 \mathrm{~nm}$ and $L_{c}=50 \mathrm{~nm}$ on the whole Y-splitter. Mean transmission to each branch with LER is $46.5 \%$ with standard deviation of $4.2 \%$ at $\lambda=1550 \mathrm{~nm}$. (a) Upper port. (b) Lower port.

To accomplish spatial sensitivity analysis we run additional simulations seeking to identify the spatial region in the Y-splitter that has the highest contribution to the resulting imbalance between the two output ports. We divide the Y-splitter into three main regions: the input taper (region 1), the junction (region 2), and the two Y-splitter arms (region 3) as shown in Fig. 1a. LER is applied to each of these regions separately as shown in Fig. 3 and for each case, we generate 50 different instantiations for every $A$ and $L_{c}$ combination to capture the transmission statistics with respect to the spatial LER. We compare the transmission imbalance caused by each section separately to the case when LER is applied to the entire structure.

When LER is imposed on the entire Y-splitter, the maximum imbalance percentage (the difference in the transmitted power between the upper are the lower output ports, normalized to the input port power) goes from $2 \%$ for small $A$ and $L_{c}$ values and can reach values of $15 \%$ for the cases when $A$ and $L_{c}$ are large, as shown in Fig. 4a. The statistics for running an ensemble of 50 different instantiations shows that the standard deviation $(\sigma)$ for the transmission imbalance can be as high as $6 \%$ for large $A$ and $L_{c}$ values and goes as low as $0.5 \%$ for small $A$ and $L_{c}$ values, as shown in Fig. 4b. 


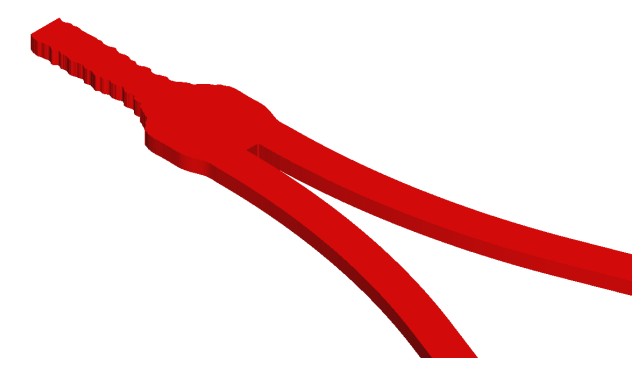

(a)

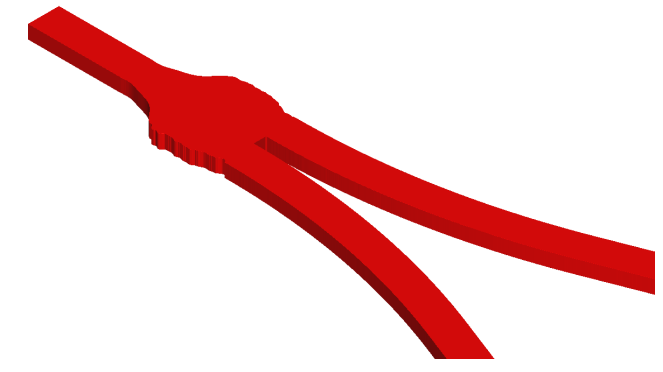

(b)

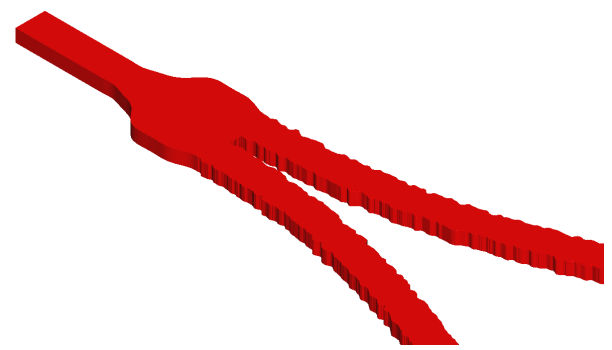

(c)

Figure 3: LER applied to different sections of the Y-splitter while performing spatial sensitivity analysis, (a) region 1: input taper, (b) region 2: junction, (c) regions 3: arms.

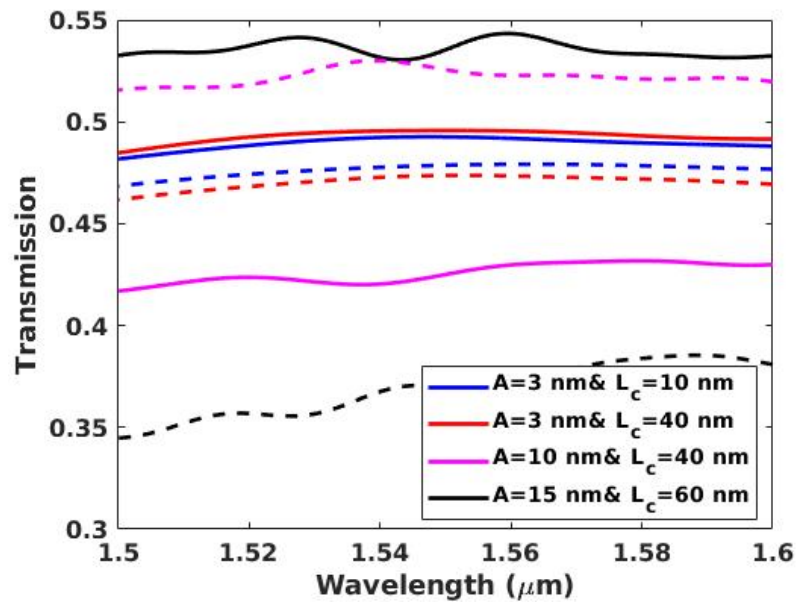

(a)

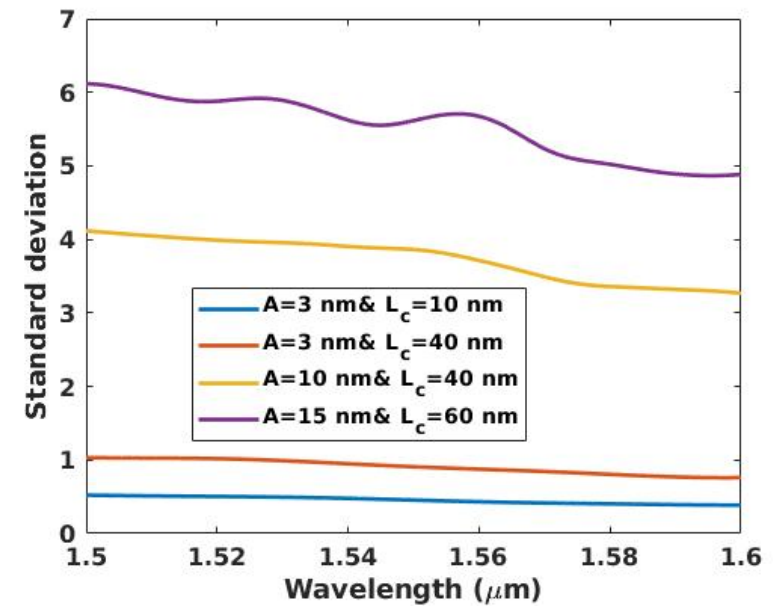

(b)

Figure 4: Transmission variation impact due to LER applied to the entire Y-splitter, based on ensembles of 50 runs for each combination of $A$ and $L_{c}$. (a) Worst case difference in normalized transmission for the two output ports (solid line is the upper port and dashed line is the lower port) from the 50 ensembles at each $A$ and $L_{c}$ combination. (b) Standard deviation $\sigma$ (in percent) of transmission imbalance calculated from the 50 run ensemble at each $A$ and $L_{c}$ combination. 


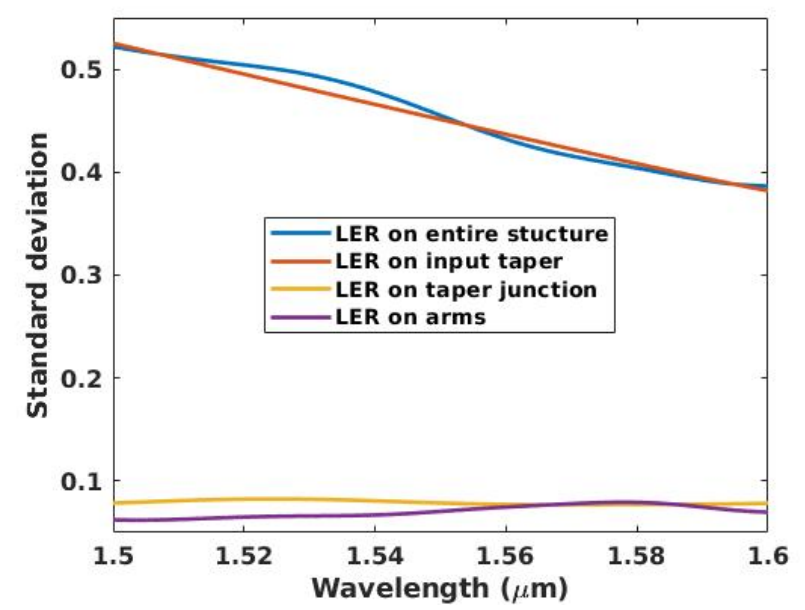

(a) $A=3 \mathrm{~nm}$ and $L_{c}=10 \mathrm{~nm}$

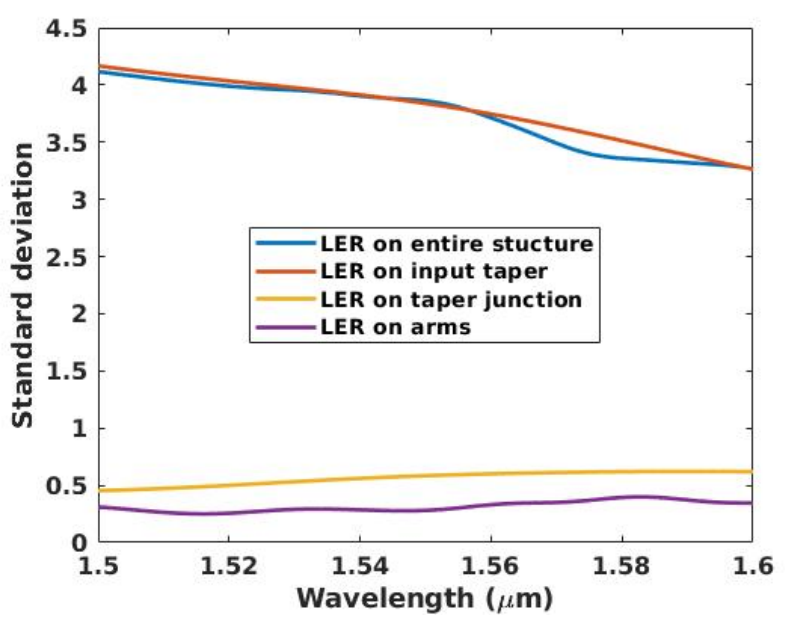

(c) $A=10 \mathrm{~nm}$ and $L_{c}=40 \mathrm{~nm}$

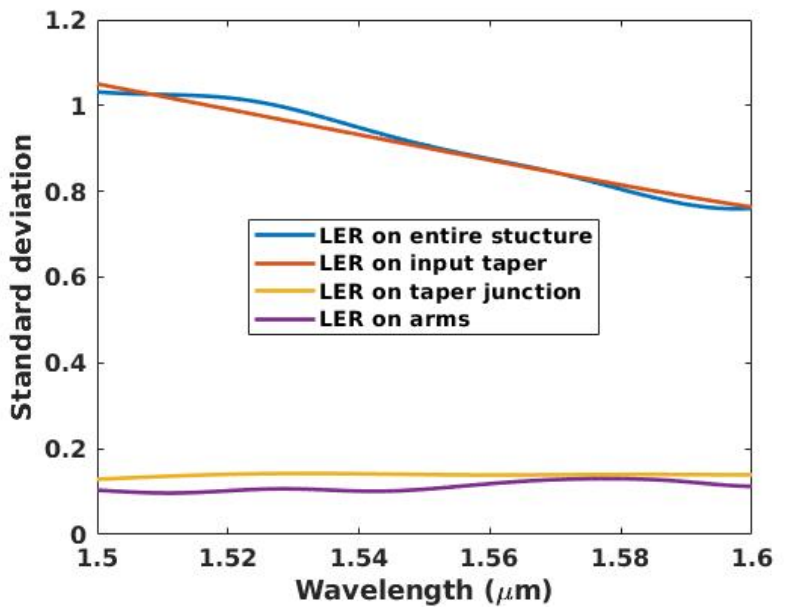

(b) $A=3 \mathrm{~nm}$ and $L_{c}=40 \mathrm{~nm}$

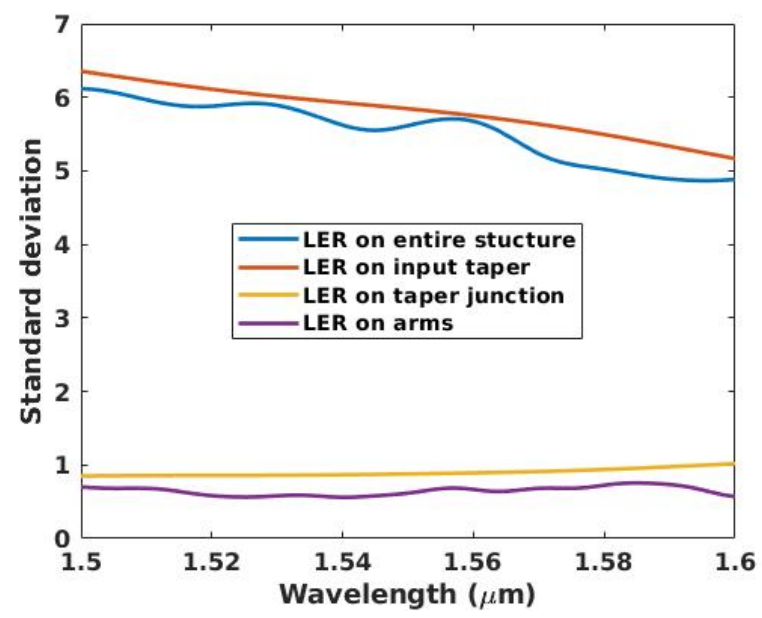

(d) $A=15 \mathrm{~nm}$ and $L_{c}=60 \mathrm{~nm}$

Figure 5: Standard deviation $\sigma$ (in percent) of the transmission imbalance caused by applying LER for different sections of the Y-splitter for different $A$ and $L_{c}$ combinations.

We next consider localized application of LER to selected regions of the Y-splitter. Fig. 5 shows the results of the standard deviation $(\sigma)$ for the power imbalance between the two output ports when the LER is applied to either the entire Y-splitter, the input taper, the junction, or the arms for each $A$ and $L_{c}$ combination. The arms seem to have the least effect ( $\sigma$ deviation from the value when there is no LER applied), indicating that LER applied only to the arms contributes relatively little to the imbalance, compared to when LER is applied to the entire device. The junction also causes small deviations on the order of those caused by the arms. However, both are much smaller than the case when LER is applied to entire structure, and therefore neither is capable of fully explaining the imbalance observed when LER is applied to the entire Y-splitter. We see that when LER is applied only to the input taper, the transmission imbalance is the largest, and is nearly equal to the values when LER is applied to the entire Y-splitter. Accordingly, this demonstrates that the Y-splitter is most sensitive to the geometric perturbations introduced by LER at the input taper, and the taper is responsible for almost all of the imbalance caused by LER in this Y-splitter design. 
Table 1: Summary of imbalanced transmission standard deviation (in percent) for LER applied to different sections of a Y-splitter at a wavelength of $1550 \mathrm{~nm}$. The values in the brackets are the $95 \%$ lower and upper bound confidence intervals calculated for the ensemble of 50 simulations.

\begin{tabular}{|c|c|c|c|c|c|c|c|c|c|}
\hline \multirow{2}{*}{$A(\mathrm{~nm})$} & \multirow{2}{*}{$L_{c}(\mathrm{~nm})$} & \multicolumn{2}{|c|}{ Entire structure } & \multicolumn{2}{|c|}{ Input taper } & \multicolumn{2}{|c|}{ Junction } & \multicolumn{2}{|c|}{ Arms } \\
\hline & & Ensemble & Adjoint & Ensemble & Adjoint & Ensemble & Adjoint & Ensemble & Adjoint \\
\hline 1 & 25 & $0.25\left(\begin{array}{l}0.31 \\
0.21\end{array}\right)$ & 0.30 & $0.25\left(\begin{array}{l}0.31 \\
0.21\end{array}\right)$ & 0.29 & $0.04\left(\begin{array}{l}0.05 \\
0.04\end{array}\right)$ & 0.05 & $0.04\left(\begin{array}{c}0.05 \\
0.04\end{array}\right)$ & 0.06 \\
\hline 3 & 10 & $0.45\left(\begin{array}{l}0.56 \\
0.38\end{array}\right)$ & 0.58 & $0.45\left(\begin{array}{l}0.56 \\
0.38\end{array}\right)$ & 0.56 & $0.08\left(\begin{array}{l}0.10 \\
0.07\end{array}\right)$ & 0.11 & $0.07\left(\begin{array}{c}0.09 \\
0.06\end{array}\right)$ & 0.11 \\
\hline 3 & 20 & $0.64\left(\begin{array}{l}0.79 \\
0.53\end{array}\right)$ & 0.82 & $0.63\left(\begin{array}{c}0.79 \\
0.53\end{array}\right)$ & 0.79 & $0.12\left(\begin{array}{l}0.15 \\
0.10\end{array}\right)$ & 0.15 & $0.09\left(\begin{array}{l}0.11 \\
0.08\end{array}\right)$ & 0.15 \\
\hline 3 & 40 & $0.90\left(\begin{array}{l}1.12 \\
0.75\end{array}\right)$ & 1.15 & $0.90\left(\begin{array}{l}1.12 \\
0.75\end{array}\right)$ & 1.11 & $0.14\left(\begin{array}{l}0.18 \\
0.12\end{array}\right)$ & 0.19 & $0.11\left(\begin{array}{c}0.14 \\
0.09\end{array}\right)$ & 0.21 \\
\hline 6 & 60 & $2.71\left(\begin{array}{l}3.38 \\
2.26\end{array}\right)$ & 2.79 & $2.59\left(\begin{array}{l}3.24 \\
2.18\end{array}\right)$ & 2.71 & $0.29\left(\begin{array}{c}0.36 \\
0.24\end{array}\right)$ & 0.44 & $0.25\left(\begin{array}{c}0.31 \\
0.21\end{array}\right)$ & 0.49 \\
\hline 7 & 30 & $2.33\left(\begin{array}{l}2.90 \\
1.94\end{array}\right)$ & 2.33 & $2.28\left(\begin{array}{l}2.85 \\
1.92\end{array}\right)$ & 2.25 & $0.32\left(\begin{array}{l}0.40 \\
0.27\end{array}\right)$ & 0.40 & $0.21\left(\begin{array}{l}0.26 \\
0.18\end{array}\right)$ & 0.43 \\
\hline 10 & 10 & $1.86\left(\begin{array}{l}2.31 \\
1.51\end{array}\right)$ & 1.93 & $1.86\left(\begin{array}{l}2.31 \\
1.51\end{array}\right)$ & 1.86 & $0.32\left(\begin{array}{c}0.40 \\
0.27\end{array}\right)$ & 0.36 & $0.18\left(\begin{array}{c}0.23 \\
0.15\end{array}\right)$ & 0.37 \\
\hline 10 & 40 & $3.85\left(\begin{array}{l}4.80 \\
3.23\end{array}\right)$ & 3.83 & $3.83\left(\begin{array}{l}4.79 \\
3.22\end{array}\right)$ & 3.70 & $0.59\left(\begin{array}{l}0.74 \\
0.50\end{array}\right)$ & 0.64 & $0.29\left(\begin{array}{c}0.36 \\
0.24\end{array}\right)$ & 0.70 \\
\hline 12 & 50 & $4.04\left(\begin{array}{l}5.05 \\
3.39\end{array}\right)$ & 5.11 & $4.09\left(\begin{array}{l}5.11 \\
3.43\end{array}\right)$ & 4.96 & $0.64\left(\begin{array}{c}0.80 \\
0.54\end{array}\right)$ & 0.83 & $0.41\left(\begin{array}{c}0.51 \\
0.34\end{array}\right)$ & 0.92 \\
\hline 15 & 60 & $5.65\left(\begin{array}{l}7.06 \\
4.75\end{array}\right)$ & 6.97 & $5.83\left(\begin{array}{c}7.29 \\
4.9\end{array}\right)$ & 6.76 & $0.88\left(\begin{array}{c}1.1 \\
0.74\end{array}\right)$ & 1.09 & $0.64\left(\begin{array}{l}0.80 \\
0.54\end{array}\right)$ & 1.23 \\
\hline
\end{tabular}

Table 1 summarizes the standard deviation (in percent) for relative output imbalance for the different $A$ and $L_{c}$ combinations with LER applied to the different regions of the Y-splitter at the operating wavelength of $1550 \mathrm{~nm}$. For all of the $A$ and $L_{c}$ combinations the input taper accounts for almost $96 \%$ of the imbalance observed when LER is applied to the entire Y-splitter.

It is worth noting that for large $A$ and $L_{c}$ combinations the input taper imbalanced transmission $\sigma$ value can be a bit more than the value when the LER is applied to the entire Y-splitter. This can be explained by considering the fact that these values are calculated using an ensemble of 50 simulations so there are confidence intervals (CI) for the calculated $\sigma$. The $95 \%$ CI shown in brackets is calculated by multiplying the $\sigma$ by 0.84 and 1.25 , respectively.

From the above simulation results we can say that the input taper (region 1) in the Y-splitter is the most sensitive section to geometrical variations (LER), and regions 2 and 3 are not as significant. These ensemble simulations (each ensemble instance consisting of virtual fabrication simulation followed by import and optical FDTD simulation to obtain the transmission spectra) are capable of providing the wavelength dependent statisti- 
cal information (mean and standard deviation) on the imbalance introduced by LER applied on different sections. However, the computational cost for running a single Y-splitter instantiation (a single run of a certain $A$ and $L_{c}$ combination) is high. For a single instance analysis for certain $A$ and $L_{c}$ combination, the total computational time from virtual fabrication, through export and import, and through optical simulation is about 200 minutes. Therefore, even in a parallel environment, this ensemble approach can be a time-consuming process.

\section{ADJOINT METHOD ANALYSIS}

Here we propose an alternative method that performs first order approximated sensitivity analysis at a much lower computational cost. The adjoint method (or adjoint state method) is a numerical method for efficiently computing the gradient of a function in a numerical optimization problem or for sensitivity analysis. ${ }^{15,16}$ It has several different forms in multiple fields of application; here the matrix form for linear system analysis is used to summarize the key ideas of the approach. ${ }^{17}$

Suppose we aim to find the derivative of the output $y(p)$ of a linear system with variation parameters $p$ :

$$
\begin{gathered}
A(p) x(p)=b \\
y(p)=c^{T} x(p) .
\end{gathered}
$$

Then from (2) and (3) it is easy to find that

$$
\frac{\mathrm{d} y(p)}{\mathrm{d} p}=-c^{T} A(p)^{-1} \frac{\mathrm{d} A(p)}{\mathrm{d} p} x(p)
$$

Thus the derivative at certain point $p_{0}$ can be computed using a direct simulation $A\left(p_{0}\right) x\left(p_{0}\right)=b$ and an adjoint simulation $A\left(p_{0}\right)^{T} v=c$, and the derivatives are connected to the solutions $x\left(p_{0}\right)$ (from direct simulation) and $v$ (from adjoint simulation) by

$$
\left.\frac{\mathrm{d} y}{\mathrm{~d} p}\right|_{p_{0}}=-\left.v^{T} \frac{\mathrm{d} A}{\mathrm{~d} p}\right|_{p_{0}} x\left(p_{0}\right) .
$$

If there are multiple variation parameters $p_{i}$, then the formula goes to

$$
\left.\frac{\mathrm{d} y}{\mathrm{~d} p_{i}}\right|_{p_{0}}=-\left.v^{T} \frac{\mathrm{d} A}{\mathrm{~d} p_{i}}\right|_{p_{0}} x\left(p_{0}\right)
$$

while $v$ and $x\left(p_{0}\right)$ maintain the same values from the direct and adjoint simulations, which means two simulations are enough to compute the derivatives with respect to all the variation parameters $p_{i}$.

The same idea has been applied to photonics design based on Maxwell's equations, enabling computation of the output derivatives with respect to any arbitrary refractive index changes with only two simulations. ${ }^{18,19}$ For photonic devices specifically, we have also implemented the adjoint method on the variation of S-parameters, by using the direct and reciprocal simulations: ${ }^{20}$

$$
\delta S_{i j}=\frac{\mathrm{i} \pi f}{2 s_{i} s_{j} \sqrt{N_{i} N_{j}}} \int_{\psi} \delta \varepsilon\left(\mathbf{x}^{\prime}\right) \mathbf{E}_{i}\left(\mathbf{x}^{\prime}\right) \cdot \mathbf{E}_{j}\left(\mathbf{x}^{\prime}\right) \mathrm{d}^{3} \mathbf{x}^{\prime}
$$

where we denote the source mode expansion coefficient in port $i$ and the electrical field in the $S_{i j}$ simulation as $s_{i}$ and $\mathbf{E}_{i}$, respectively; the terms $s_{j}$ and $\mathbf{E}_{j}$ analogously for $S_{j i}$; and the power of the mode in port $i$ and port $j$ as $N_{i}$ and $N_{j}$. For example, if we aim to identify the cause of transmission imbalance in a Y-splitter:

$$
\delta T=\delta\left|S_{12}\right|^{2}-\delta\left|S_{13}\right|^{2}
$$

where we label the input port as port 1 and the two output ports as port 2 and port 3, then by using (7), we can show that

$$
\delta T=\int_{\psi} R\left(\mathbf{x}^{\prime}\right) \delta n\left(\mathbf{x}^{\prime}\right) \mathrm{d}^{3} \mathbf{x}^{\prime}
$$


The refractive index sensitivity function (plane view shown in Fig. 7a) is

$$
R\left(\mathbf{x}^{\prime}\right)=-\frac{2 \pi f \varepsilon_{0}}{\left|s_{1}\right|^{2} N_{1}} \operatorname{Im} \frac{a_{12}^{*}}{s_{2}} n\left(\mathbf{x}^{\prime}\right) \mathbf{E}\left(\mathbf{x}^{\prime}\right) \cdot \mathbf{E}^{A}\left(\mathbf{x}^{\prime}\right)
$$

where $a_{12}$ is the monitor mode expansion coefficient in port 2 in the $S_{12}$ simulation, $\mathbf{E}\left(\mathbf{x}^{\prime}\right)=\mathbf{E}_{1}\left(\mathbf{x}^{\prime}\right)$ is from the direct simulation by injecting the mode from the input port (Fig. 6a), and $\mathbf{E}^{A}\left(\mathbf{x}^{\prime}\right)=\mathbf{E}_{2}\left(\mathbf{x}^{\prime}\right)-\mathbf{E}_{3}\left(\mathbf{x}^{\prime}\right)$ is from the adjoint simulation by sending the out-of-phase modes backward from the two output ports (Fig. 6b).
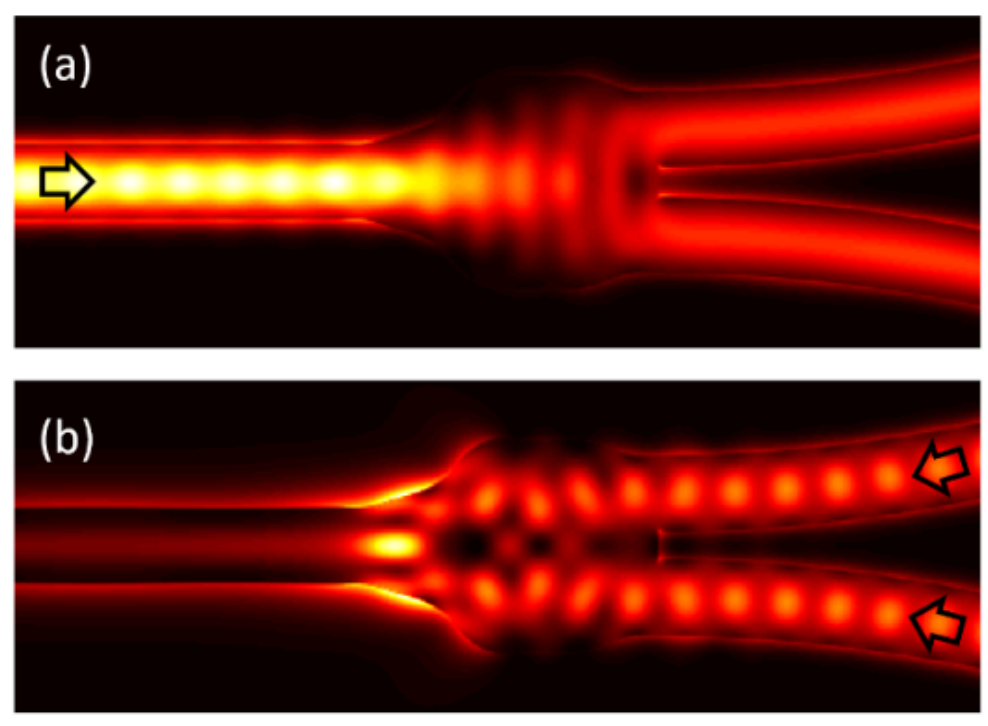

Figure 6: Intensity of the electrical field in the y-branch at the center plane of the waveguide, for (a) direct simulation $\mathbf{E}\left(\mathbf{x}^{\prime}\right)$ and (b) adjoint simulation $\mathbf{E}^{A}\left(\mathbf{x}^{\prime}\right)$. Arrows indicate the propagating direction of the mode. Operating wavelength is $1550 \mathrm{~nm}$.

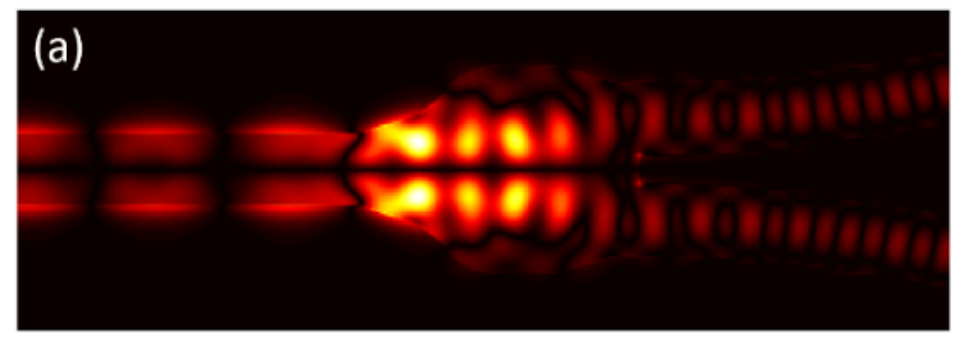

(b)

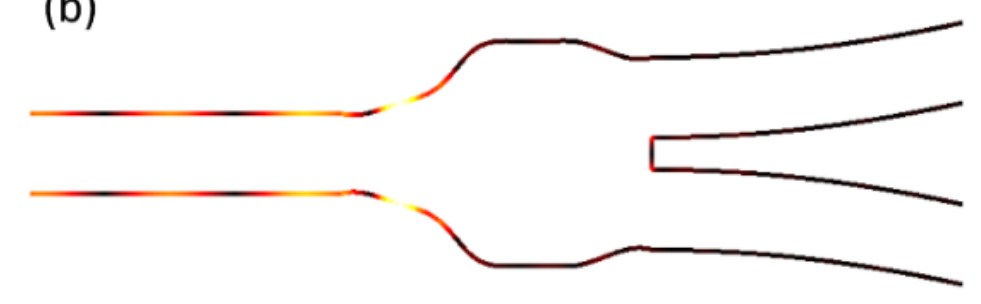

Figure 7: Adjoint method analysis results: (a) index sensitivity function $\left|R\left(\mathbf{x}^{\prime}\right)\right|$ at the center plane of the waveguide; (b) geometry sensitivity function $\left|g\left(x^{\prime}, y^{\prime}\right)\right|$ at the sidewall. 
For the shape variation specifically, perturbation theory ${ }^{21}$ is incorporated to address the discontinuity on the material interface, which gives ${ }^{20}$

$$
\delta T=\int_{C} g\left(x^{\prime}, y^{\prime}\right) \delta x_{n}^{\prime} \mathrm{d} l^{\prime}
$$

where the integral is performed on the sidewall contour $C$, and $\delta x_{n}$ is the surface variation in the normal direction. The geometry sensitivity function (shown in Fig. $7 \mathrm{~b}$ ) is

$$
g\left(x^{\prime}, y^{\prime}\right)=-\frac{\pi f \varepsilon_{0}}{\left|s_{1}\right|^{2} N_{1}} \operatorname{Im} \frac{a_{12}^{*}}{s_{2}} \int_{0}^{h}\left[\Delta\left(n^{2}\right)\left(E_{\|} \cdot E_{\|}^{A}\right)-\Delta\left(n^{-2}\right)\left(D_{\perp} \cdot D_{\perp}^{A}\right) / \varepsilon_{0}^{2}\right] \mathrm{d} z^{\prime},
$$

where $E_{\|}$and $E_{\|}^{A}$ are the parallel component of electric field, $D_{\perp}$ and $D_{\perp}^{A}$ are the perpendicular component of electric displacement field and $\Delta\left(n^{d}\right)=n_{1}^{d}-n_{2}^{d}$ is the $d$-th power index difference between silicon and the oxide cladding.

Equations (1) and (11) can be combined for estimation of the variance of the transmission imbalance caused by LER: ${ }^{20}$

$$
\sigma^{2}=A^{2} \int_{C} \int_{C} g\left(x^{\prime}, y^{\prime}\right) g\left(x^{\prime \prime}, y^{\prime \prime}\right) e^{-\left(\left|\mathbf{x}^{\prime}-\mathbf{x}^{\prime \prime}\right| / L_{c}\right)^{2}} \mathrm{~d} l^{\prime} \mathrm{d} l^{\prime \prime}
$$

When the correlation length $L_{c}$ is much smaller than the length scale at which $g\left(x^{\prime}, y^{\prime}\right)$ varies, (13) can be approximated and simplified as

$$
\sigma^{2} \approx \sqrt{\pi} A^{2} L_{c} \int_{C} g^{2}\left(x^{\prime}, y^{\prime}\right) \mathrm{d} l^{\prime} .
$$

Notice that both $\left|R\left(\mathbf{x}^{\prime}\right)\right|$ and $\left|g\left(x^{\prime}, y^{\prime}\right)\right|$ only depend on the nominal design but not the specific variation; thus for the LER imposed on different sections of the structure, the only difference in (13) or (14) is just the integral contour $C$ (Fig. 8). It is easy to identify that the sensitivities come from the input taper (defined as region 1 in Fig. 1a), which is consistent with the results from virtual fabrication simulations. Furthermore, as suggested in Fig. $6 \mathrm{~b}$, where the out-of-phase modes combine to an anti-symmetric mode in the input waveguide, the adjoint method explains the source of imbalance as the result of the anti-symmetric mode introduced by LER at the input taper region.

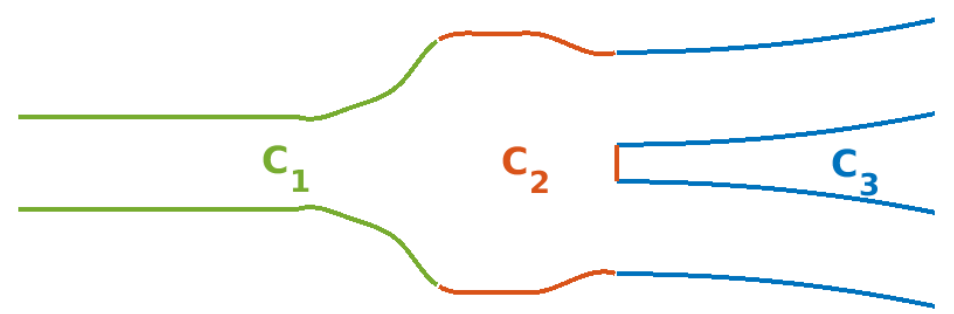

Figure 8: The integral contour for LER imposed on input taper $\left(C_{1}\right)$, junction $\left(C_{2}\right)$, and arms $\left(C_{3}\right)$, used in adjoint analysis (13) and (14).

If (14) is used, then the integral is independent of amplitude $A$ and correlation length $L_{c}$, which suggests that the ratio of contribution of LER imposed on each region of the structure to the variance of the transmission imbalance is constant throughout small perturbation amplitude and small correlation length: the input taper, junction, and arms contributes to approximately $93.5 \%, 3 \%$, and $3.5 \%$ of the variance of transmission imbalance, respectively, and $97 \%, 16 \%, 18 \%$ of the standard deviation. Notice that based on (14), the variance of the contribution of each region will sum up to the variance of the entire structure, but not the standard deviation. This is also observed in Table 1.

Table 1 also summarizes the standard deviation $(\sigma)$ for relative output imbalance for the different $A$ and $L_{c}$ combinations with LER applied to the different regions of the Y-splitter at the operating wavelength of $1550 \mathrm{~nm}$, estimated from the adjoint method. For the entire structure, as discussed in ${ }^{20}$ the two approaches only show 
good consistency for LER amplitude $6 \mathrm{~nm} \leq A \leq 10 \mathrm{~nm}$. For large LER amplitude $A>10 \mathrm{~nm}$, the adjoint method begins to hit the limits of its first order approximation for geometry perturbations, and the FDTD simulation is expected to be more accurate. However, for small LER amplitudes, FDTD simulation used in the ensemble approach confronts meshing difficulty to capture the details of very small geometric changes, and thus for LER amplitudes, $A<6 \mathrm{~nm}$, the adjoint method is expected to be more accurate than ensemble simulation. Examining the contributions of each region in the comparable middle amplitude range, the differences between the two approaches are very small for the input taper region, but become significant for the junction and arm regions. While the wide confidence interval from the ensemble approach which estimates the standard deviation from only 50 random samples may explain part of the differences, it is likely that the numerical error in calculating the geometry sensitivity function $g\left(x^{\prime}, y^{\prime}\right)$ becomes significant in these two regions, where the absolute value of the function is already close to zero (see Fig. 6b). This also explains why the differences are even larger for the arm region, where the integral contour is much longer than that of the junction region.

Using the adjoint approach, the computational cost is much reduced compared to the ensemble approach because time-consuming virtual fabrication is not involved (only the nominal device geometry is required), and there is no need to run a full ensemble of simulations. The total computational time from direct and adjoint simulations and the numerical analysis is about 80 minutes. One issue in the adjoint approach is the memory cost for the storage of the electrical field, which is around 1.5 GB for a single wavelength. Thus the adjoint analysis can only be performed at selected frequency points, whereas the simulated based method uses a much finer frequency grid of 1001 points.

\section{CONCLUSION}

In summary, we have performed a sensitivity analysis study to identify the most sensitive regions within a Ysplitter to line edge roughness (LER) using two complementary methods: a simulation based method (ensemble of virtual fabrication simulations) and a numerically based method (adjoint analysis). The results of both studies are consistent and reveal that the most sensitive region in the Y-splitter is the input taper where the power starts to split between the two output ports, so any small variation in the geometry that breaks the symmetry in this region results in imbalanced transmission. From the computational cost point of view, the ensemble simulation based analysis takes more time than the adjoint analysis. However, the simulation based analysis gives higher precision results on a finer frequency grid; the adjoint method is a first order approximation and can only be performed at a relatively small number of frequency points. Thus there is a trade off between the amount of data gained and the computational cost paid.

Both methodologies can be extended to different photonic components and can be used to check sensitivity against various process variations. The choice of the method is application dependent. For instance, one may care about identifying the most sensitive section of a component, or about quantitatively studying effects across a wide frequency spectrum in applications where high precision designs are needed. The results from this sensitivity analysis, and others, when included in an extended process design kit (PDK) would help photonic designers predict and optimize photonic behavior and yield. Such variation-aware design methods contribute toward the goal of high yield silicon photonics manufacturing.

\section{ACKNOWLEDGMENTS}

This material is based on research sponsored in part by AIM Photonics and Air Force Research Laboratory under agreement number FA8650-15-2-5220. The U.S. Government is authorized to reproduce and distribute reprints for Governmental purposes notwithstanding any copyright notation thereon. The views and conclusions contained herein are those of the authors and should not be interpreted as necessarily representing the official policies or endorsements, either expressed or implied, of Air Force Research Laboratory or the U.S. Government.

\section{REFERENCES}

[1] Chrostowski, L. and Hochberg, M., [Silicon Photonics Design: From Devices to Systems], Cambridge University Press (2015). 
[2] Wang, X., Shi, W., Yun, H., Grist, S., Jaeger, N. A., and Chrostowski, L., "Narrow-band waveguide Bragg gratings on SOI wafers with CMOS-compatible fabrication process," Optics Express 20(14), 15547-15558 (2012).

[3] Sun, C., Wade, M. T., Lee, Y., Orcutt, J. S., Alloatti, L., Georgas, M. S., Waterman, A. S., Shainline, J. M., Avizienis, R. R., Lin, S., et al., "Single-chip microprocessor that communicates directly using light," Nature 528(7583), 534 (2015).

[4] Bowers, J. E., Komljenovic, T., Davenport, M., Hulme, J., Liu, A. Y., Santis, C. T., Spott, A., Srinivasan, S., Stanton, E. J., and Zhang, C., "Recent advances in silicon photonic integrated circuits," in [Next-Generation Optical Communication: Components, Sub-Systems, and Systems V], 9774, 977402, International Society for Optics and Photonics (2016).

[5] Zortman, W. A., Trotter, D. C., and Watts, M. R., "Silicon photonics manufacturing," Optics Express 18(23), 23598 (2010).

[6] El-Henawy, S. I., Miller, R., and Boning, D. S., "Effects of a random process variation on the transfer characteristics of a fundamental photonic integrated circuit component," in [Proc. of SPIE], 10743, 107430O-1-107430O-10 (2018).

[7] Wu, R., Chen, C.-H., Huang, T.-C., Beausoleil, R., and Cheng, K.-T., "Spatial pattern analysis of process variations in silicon microring modulators," in [IEEE Optical Interconnects Conf.], 116-117 (2016).

[8] Melati, D., Melloni, A., and Morichetti, F., "Real photonic waveguides: guiding light through imperfections," Advances in Optics and Photonics 6(2), 156-224 (2014).

[9] Shin, C. et al., [Variation-aware advanced CMOS devices and SRAM], vol. 56, Springer (2016).

[10] Zhang, Y., Yang, S., Lim, A. E.-J., Lo, G.-Q., Galland, C., Baehr-Jones, T., and Hochberg, M., "A compact and low loss Y-junction for submicron silicon waveguide," Optics Express 21(1), 1310-1316 (2013).

[11] Available:. https://www . coventor.com/semiconductor-solutions/semulator3d.

[12] Asenov, A., Kaya, S., and Brown, A. R., "Intrinsic parameter fluctuations in decananometer MOSFETs introduced by gate line edge roughness," IEEE Trans. on Electron Devices 50(5), 1254-1260 (2003).

[13] Mack, C. A., "Generating random rough edges, surfaces, and volumes," Applied Optics 52(7), 1472-1480 (2013).

[14] Available:. https://www.lumerical.com/products/fdtd-solutions.

[15] Errico, R. M., "What Is an Adjoint Model?," Bulletin of the American Meteorological Society 78, 2577-2591 (11 1997).

[16] Cao, Y., Li, S., Petzold, L., and Serban, R., "Adjoint sensitivity analysis for differential-algebraic equations: The adjoint DAE system and its numerical solution," SIAM Journal on Scientific Computing 24(3), 10761089 (2003).

[17] Branin, F., "Network sensitivity and noise analysis simplified," IEEE Trans. on Circuit Theory 20(3), 285-288 (1973).

[18] Lalau-Keraly, C. M., Bhargava, S., Miller, O. D., and Yablonovitch, E., "Adjoint shape optimization applied to electromagnetic design," Optics Express 21(18), 21693 (2013).

[19] Miller, O. D., "Photonic design: From fundamental solar cell physics to computational inverse design," preprint, arXiv:1308.0212 (2013).

[20] Zhang, Z., El-Henawy, S. I., Sadun, A., Miller, R., Daniel, L., White, J., and Boning, D., "Adjoint-Based sensitivity analysis for silicon photonic variations," in [IEEE MTT-S International Conference on Numerical Electromagnetic and Multiphysics Modeling and Optimization (NEMO 2019)], (May 2019).

[21] Johnson, S. G., Ibanescu, M., Skorobogatiy, M. A., Weisberg, O., Joannopoulos, J. D., and Fink, Y., "Perturbation theory for Maxwell's equations with shifting material boundaries," Phys. Rev. E 65, 066611 (June 2002). 